Supporting Information

\title{
Role of Dynamical Electron Correlation in the Differences in Bonding Between $\mathrm{CaAlH}_{3}$ and $\mathrm{MgAlH}_{3}$
}

Fabio E. Penotti ${ }^{\dagger}$, David L. Cooper ${ }^{\ddagger}$ and Peter B. Karadakov§

${ }^{\dagger}$ Consiglio Nazionale delle Ricerche, Istituto di Scienze e Tecnologie Chimiche "Giulio Natta", Via Golgi 19, I-20133 Milano MI, Italy

‡Department of Chemistry, University of Liverpool, Liverpool L69 7ZD, UK

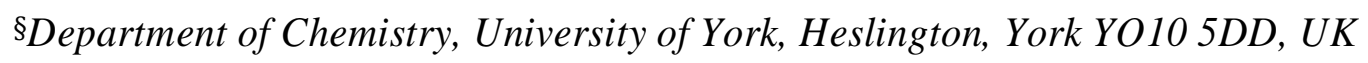

Corresponding authors: Fabio E. Penotti - Email: f.penotti@gmail.com; David L. Cooper - Email: dlc@liverpool.ac.uk 


\section{$\underline{\text { Table of Contents }}$}

Table S1 $\quad$ CCSD(T) energies for various geometries of $\mathrm{M}^{\prime} \mathrm{AlH} 3$

Table S2 CCSD(T) energies for symmetrically distorted $\mathrm{AlH}_{3} \quad$ S3

Table S3 $\quad$ CCSD atomic populations for $\mathrm{M}^{\prime} \mathrm{AlH}_{3} \quad \mathrm{~S} 4$

Table S4 $\quad \mathrm{M}^{\prime}$....H3 Coulombic interaction energies for $\mathrm{M}^{\prime} \mathrm{AlH}_{3} \quad$ S4

Table S5 $\quad \mathrm{M}^{\prime}$....AlH3 Coulombic interaction energies for $\mathrm{M}^{\prime} \mathrm{AlH}_{3} \quad$ S5

Table S6 RHF, SCGVB and CASSCF energies for $\mathrm{M}^{\prime} \mathrm{AlH}_{3} \quad$ S5

Table S7 SCGVB orbital overlaps for $\mathrm{MgAlH}$ at its model $\theta=-35^{\circ}$ geometry $\quad$ S6

Table S8 SCGVB orbital overlaps for $\mathrm{CaAlH}$ at its model $\theta=+4^{\circ}$ geometry S6

Figure S1 Symmetry-unique SCGVB orbitals at model geometries S6

Table S9 Simple energy differences between $\theta \sim-35^{\circ}$ and $\theta \sim+4^{\circ}$ geometries $\quad$ S7 
Table S1 CCSD(T) energies (in hartree) for various $\mathrm{C}_{3 v}$ geometries of $\mathrm{MgAlH}_{3}$ and $\mathrm{CaAlH}_{3}$.

\begin{tabular}{cccccc}
\hline & $R_{1} / \AA$ & $R_{2} / \AA$ & $\theta$ & Energy & $R_{\mathrm{M}^{\prime} \mathrm{H}} / \AA$ \\
\hline MgAlH3 & & & & & \\
aug-cc-pVTZ & 2.952711 & 1.589493 & $+3.92063^{\circ}$ & -443.5185795 & 3.447724 \\
& 2.952711 & 1.589493 & $-35^{\circ}$ & -443.4809235 & 2.420959 \\
& 2.434514 & 1.589493 & $-35^{\circ}$ & -443.5056371 & 2.003565 \\
& 2.439984 & 1.692880 & $-35^{\circ}$ & -443.5114212 & 2.020133 \\
aug-cc-pVQZ & 3.010287 & 1.560233 & $+3.46140^{\circ}$ & -443.5302167 & 3.473226 \\
& 2.379944 & 1.640973 & $-35^{\circ}$ & -443.5228466 & 1.968961
\end{tabular}

$\mathrm{CaAlH}_{3}$

\begin{tabular}{cccccc} 
aug-cc-pVTZ & 2.749063 & 1.696520 & $-34.80580^{\circ}$ & -920.8255329 & 2.260823 \\
& 2.749063 & 1.696520 & $+4^{\circ}$ & -920.7915749 & 3.329594 \\
& 3.243867 & 1.696520 & $+4^{\circ}$ & -920.8021233 & 3.764125 \\
& 3.279750 & 1.592916 & $+4^{\circ}$ & -920.8087955 & 3.744731 \\
aug-cc-pVQZ & 2.655714 & 1.642523 & $-36.73782^{\circ}$ & -920.8791522 & 2.128923 \\
& 3.174663 & 1.563659 & $+4^{\circ}$ & -920.8455061 & 3.635391 \\
\hline
\end{tabular}

Table S2 CCSD(T)/aug-cc-pVQZ energies (in hartree) and relative energies $\Delta E_{\mathrm{d}}$ (in $\mathrm{kcal} / \mathrm{mol}$ ) for symmetrically distorted $\mathrm{AlH}$.

\begin{tabular}{cccc}
\hline$\theta$ & $R_{\mathrm{AlH}} / \AA$ & Energy & $\Delta E_{\mathrm{d}}$ \\
\hline $0^{\circ}$ & 1.55301308 & -243.8378853 & 0 \\
$3.46139925^{\circ}$ & 1.56023279 & -243.8369119 & 0.61 \\
$4^{\circ}$ & 1.56365923 & -243.8365491 & 0.84 \\
$35^{\circ}$ & 1.64097258 & -243.7432534 & 59.38 \\
$36.73781912^{\circ}$ & 1.64252250 & -243.7334681 & 65.52 \\
$0^{\circ}$ & 1.56023279 & -243.8378360 & 0.03 \\
$0^{\circ}$ & 1.56365923 & -243.8377780 & 0.07 \\
$0^{\circ}$ & 1.64097258 & -243.8312644 & 4.15 \\
$0^{\circ}$ & 1.64252250 & -243.8310430 & 4.29 \\
\hline
\end{tabular}


Table S3 CCSD atomic populations at the CCSD(T)/aug-cc-pVQZ C3v geometries of M'AlH3 (see Table S1), except for values taken from Ref. 1.

\begin{tabular}{|c|c|c|c|c|c|c|c|c|}
\hline \multirow{3}{*}{ Populations } & \multicolumn{4}{|c|}{$\theta \sim+4^{\circ}$} & \multicolumn{4}{|c|}{$\theta \sim-35^{\circ}$} \\
\hline & \multicolumn{2}{|c|}{$\mathrm{MgAlH}_{3}$} & \multicolumn{2}{|c|}{$\mathrm{CaAlH}_{3}$} & \multicolumn{2}{|c|}{$\mathrm{MgAlH}_{3}$} & \multicolumn{2}{|c|}{$\mathrm{CaAlH}_{3}$} \\
\hline & $\mathrm{Mg}$ & $\mathrm{Al}$ & $\mathrm{Ca}$ & $\mathrm{Al}$ & Mg & $\mathrm{Al}$ & $\mathrm{Ca}$ & $\mathrm{Al}$ \\
\hline Ref. 1 & 0.300 & 0.854 & & & & & 0.975 & 0.453 \\
\hline \multicolumn{9}{|l|}{ aug-cc-pVQZ } \\
\hline NPA & 0.293 & 0.883 & 0.462 & 0.693 & 1.002 & 0.444 & 1.178 & 0.385 \\
\hline VDD & 0.206 & 0.379 & 0.272 & 0.323 & 0.341 & 0.212 & 0.397 & 0.139 \\
\hline \multicolumn{9}{|l|}{$6-31 G^{* *}$} \\
\hline NPA & 0.281 & 0.832 & 0.401 & 0.680 & 0.945 & 0.427 & 1.034 & 0.467 \\
\hline VDD & 0.191 & 0.377 & 0.247 & 0.330 & 0.313 & 0.217 & 0.335 & 0.179 \\
\hline Mulliken & 0.103 & 0.437 & 0.146 & 0.391 & 0.180 & 0.331 & 0.363 & 0.365 \\
\hline
\end{tabular}

Table S4 $\quad \mathrm{M}^{\prime} \ldots \mathrm{H}_{3}$ Coulombic interaction energies (in eV) at the $C_{3 v} \operatorname{CCSD}(\mathrm{T}) /$ aug-cc-pVQZ geometries of $\mathrm{M}^{\prime} \mathrm{AlH}_{3}$ (see Table S1) calculated using CCSD atomic populations (see Table S3), except for the values taken from Ref. 1.

\begin{tabular}{rrrrrr}
\hline \multirow{2}{*}{ Populations } & \multicolumn{2}{c}{$\theta \sim+4^{\circ}$} & & \multicolumn{2}{c}{$\theta \sim-35^{\circ}$} \\
\cline { 2 - 3 } \cline { 5 - 6 } Ref. 1 & -1.43 & & & -8.96 \\
\hline MgAlH3 & $\mathrm{CaAlH}_{3}$ & & $\mathrm{MgAlH}_{3}$ & $\mathrm{CaAlH} 3$ \\
NPA-cc-pVQZ & & & & & \\
VDD & -0.50 & -0.64 & & -1.38 & -1.44 \\
6-31G** & & & & & \\
NPA & -1.29 & -1.72 & & -9.48 & -10.49 \\
VDD & -0.45 & -0.56 & & -1.22 & -1.16 \\
Mulliken & -0.23 & -0.31 & & -0.67 & -1.79 \\
\hline
\end{tabular}


Table S5 $\quad \mathrm{M}^{\prime}$...AlH 3 Coulombic interaction energies (in $\mathrm{eV}$ ) at the $C_{3 v} \mathrm{CCSD}(\mathrm{T})$ /aug-cc-pVQZ geometries of $\mathrm{M}^{\prime} \mathrm{AlH}_{3}$ (see Table S1) calculated using CCSD atomic populations (Table S3), except for the values based on data in Ref. 1.

\begin{tabular}{rccccc}
\hline \multirow{2}{*}{ Populations } & \multicolumn{2}{c}{$\theta \sim+4^{\circ}$} & & \multicolumn{2}{c}{$\theta \sim-35^{\circ}$} \\
\cline { 2 - 3 } \cline { 5 - 6 } \cline { 5 - 6 } \cline { 5 - 6 } Ref. 1 & -0.20 & & & & -6.63 \\
MgAlH3 & $\mathrm{CaAlH}_{3}$ & & & & \\
aug-cc-pVQZ & & & & & \\
NPA & -0.19 & -0.66 & & -7.90 & -10.00 \\
VDD & -0.13 & -0.24 & & -0.94 & -1.14 \\
6-31G** & & & & & \\
NPA & -0.18 & -0.48 & & -7.04 & -7.87 \\
VDD & -0.11 & -0.19 & & -0.80 & -0.84 \\
Mulliken & -0.02 & -0.05 & & -0.31 & -1.07 \\
\hline
\end{tabular}

Table S6 RHF, SCGVB and CASSCF energies (in hartree) at the aug-cc-pVQZ $C_{3 v}$ geometries of $\mathrm{M}^{\prime} \mathrm{AlH}$ (see Table S1). (Proportions of corresponding CASSCF $(8,8)$ electron correlation energy are shown as percentages.)

\begin{tabular}{|c|c|c|c|c|}
\hline \multirow{2}{*}{ Method } & \multicolumn{2}{|c|}{$\theta \sim+4^{\circ}$} & \multicolumn{2}{|c|}{$\theta \sim-35^{\circ}$} \\
\hline & $\mathrm{MgAlH}_{3}$ & $\mathrm{CaAlH}_{3}$ & $\mathrm{MgAlH}_{3}$ & $\mathrm{CaAlH}_{3}$ \\
\hline RHF & -443.2584671 & -920.3973239 & -443.2087862 & -920.3778982 \\
\hline \multirow[t]{2}{*}{$\mathrm{SCGVB}^{a}$} & -443.3156902 & -920.4584124 & -443.2911489 & -920.4572730 \\
\hline & $(98.9 \%)$ & (98.9\%) & (98.7\%) & $(98.9 \%)$ \\
\hline $\mathrm{SCGVB}^{b}$ & -443.3156652 & -920.4583892 & -443.2911410 & -920.4572505 \\
\hline CASSCF $(8,8)$ & -443.3163154 & -920.4591111 & -443.2922291 & -920.4581244 \\
\hline CASSCF $(8,11)$ & -443.3407315 & -920.4835907 & -443.3231489 & -920.4866732 \\
\hline
\end{tabular}

${ }^{a}$ Inactive orbitals taken from CASSCF $(8,8)$

${ }^{b}$ Inactive orbitals taken from CASSCF $(8,11)$ 
Table S7 SCGVB orbital overlaps for $\mathrm{MgAlH}_{3}$ at its model $\theta=-35^{\circ}$ geometry.

\begin{tabular}{c|cccccccc|}
\multicolumn{1}{c}{} & $\varphi 1$ & $\varphi 2$ & $\varphi 3$ & $\varphi 4$ & $\varphi 5$ & $\varphi 6$ & $\varphi 7$ & $\varphi 8$ \\
\cline { 2 - 9 }$\varphi_{1}$ & 1 & & & & & & & \\
$\varphi_{2}$ & 0.83 & 1 & & & & & & \\
$\varphi_{3}$ & 0.13 & 0.20 & 1 & & & & & \\
$\varphi_{4}$ & 0.20 & 0.33 & 0.83 & 1 & & & & \\
$\varphi_{5}$ & 0.13 & 0.20 & 0.13 & 0.20 & 1 & & & \\
$\varphi_{6}$ & 0.20 & 0.33 & 0.20 & 0.33 & 0.83 & 1 & & \\
$\varphi_{7}$ & 0.12 & 0.29 & 0.12 & 0.29 & 0.12 & 0.29 & 1 & \\
$\varphi_{8}$ & 0.10 & 0.13 & 0.10 & 0.13 & 0.10 & 0.13 & -0.25 & 1 \\
\cline { 2 - 9 }
\end{tabular}

Table S8 SCGVB orbital overlaps for $\mathrm{CaAlH}$ 3 at its model $\theta=+4^{\circ}$ geometry.

\begin{tabular}{c|cccccccc|}
\multicolumn{1}{c}{} & $\varphi_{1}$ & $\varphi_{2}$ & $\varphi_{3}$ & $\varphi_{4}$ & $\varphi_{5}$ & $\varphi_{6}$ & $\varphi_{7}$ & $\varphi 8$ \\
\cline { 2 - 9 }$\varphi_{1}$ & 1 & & & & & & & \\
$\varphi_{2}$ & 0.81 & 1 & & & & & & \\
$\varphi_{3}$ & 0.07 & 0.14 & 1 & & & & & \\
$\varphi_{4}$ & 0.14 & 0.30 & 0.81 & 1 & & & & \\
$\varphi_{5}$ & 0.07 & 0.14 & 0.07 & 0.14 & 1 & & & \\
$\varphi_{6}$ & 0.14 & 0.30 & 0.14 & 0.30 & 0.81 & 1 & & \\
$\varphi_{7}$ & 0.14 & 0.28 & 0.14 & 0.28 & 0.14 & 0.28 & 1 & \\
$\varphi_{8}$ & 0.02 & 0.03 & 0.02 & 0.03 & 0.02 & 0.03 & 0.48 & 1 \\
\cline { 2 - 8 } & & & & & & & &
\end{tabular}

Figure S1 Symmetry-unique SCGVB orbitals for MgAlHз (top row) and $\mathrm{CaAlH}_{3}$ (bottom row) at model geometries.

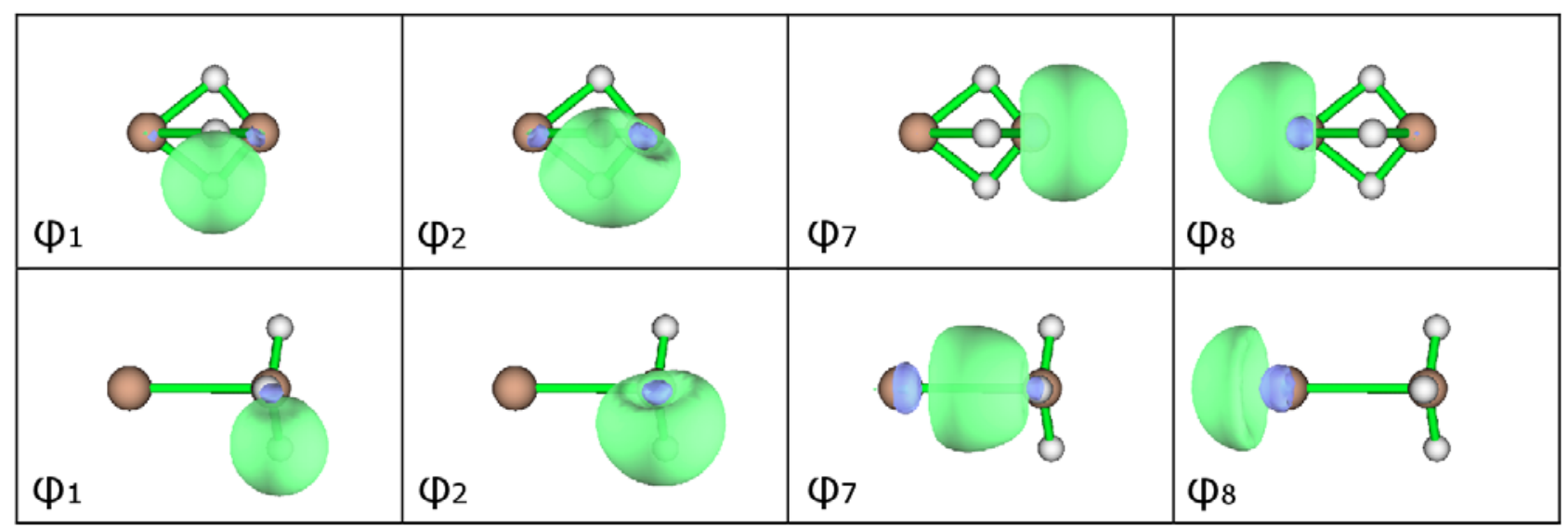


Table S9 Simple energy differences between the $\theta \sim-35^{\circ}$ and $\theta \sim+4^{\circ} \mathrm{CCSD}(\mathrm{T}) /$ aug-cc-pVQZ geometries, as calculated for a given molecule at various levels of theory using the aug-cc-pVQZ basis set. Negative values of $\Delta E$ indicate a preference for the $\theta \sim-35^{\circ}$ geometry.

\begin{tabular}{ccc}
\hline Method & \multicolumn{2}{c}{$\Delta E$ (in kcal/mol) } \\
\cline { 2 - 3 } RHF & MgAlH3 & $\mathrm{CaAlH}_{3}$ \\
\cline { 2 - 3 } SCGVB $^{a}$ & 15.4 & 12.2 \\
SCGVB $^{b}$ & 15.4 & 0.7 \\
CASSCF(8,8) & 15.1 & 0.6 \\
CASSCF(8,11) & 11.0 & -1.9 \\
B3LYP & 12.9 & -6.8 \\
CCSD & 9.0 & -16.6 \\
CCSD(T) & 4.6 & -21.1 \\
\hline
\end{tabular}

${ }^{a}$ Inactive orbitals taken from CASSCF $(8,8)$

${ }^{b}$ Inactive orbitals taken from CASSCF $(8,11)$ 